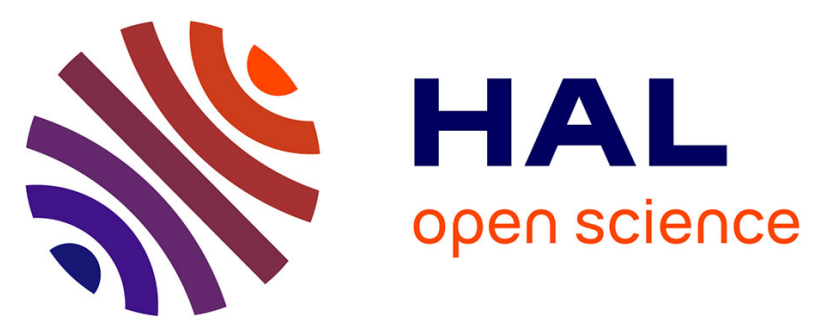

\title{
Mapping of a copper-binding site on the small CP12 chloroplastic protein of Chlamydomonas reinhardtii using top-down mass spectrometry and site-directed mutagenesis
}

Jenny Erales, Brigitte Gontero, Julian Whitelegge, Frédéric Halgand

\section{To cite this version:}

Jenny Erales, Brigitte Gontero, Julian Whitelegge, Frédéric Halgand. Mapping of a copperbinding site on the small CP12 chloroplastic protein of Chlamydomonas reinhardtii using top-down mass spectrometry and site-directed mutagenesis. Biochemical Journal, 2009, 419 (1), pp.75 - 86. 10.1042/BJ20082004 . hal-00479106

\section{HAL Id: hal-00479106 https://hal.science/hal-00479106}

Submitted on 30 Apr 2010

HAL is a multi-disciplinary open access archive for the deposit and dissemination of scientific research documents, whether they are published or not. The documents may come from teaching and research institutions in France or abroad, or from public or private research centers.
L'archive ouverte pluridisciplinaire HAL, est destinée au dépôt et à la diffusion de documents scientifiques de niveau recherche, publiés ou non, émanant des établissements d'enseignement et de recherche français ou étrangers, des laboratoires publics ou privés. 


\title{
MAPPING OF A COPPER BINDING SITE ON THE SMALL CP12 CHLOROPLASTIC PROTEIN, USING TOP DOWN MASS SPECTROMETRY AND SITE- DIRECTED MUTAGENESES.
}

\author{
Jenny Erales*, Brigitte Gontero*, Julian Whitelegge ${ }^{\dagger}$ and Frédéric Halgand ${ }^{\dagger \ddagger}$
}

From the ${ }^{*}$ Laboratoire Enzymologie des complexes supra-moléculaires; BIP (Bioénergétique et Ingénierie des Protéines) ; UPR 9036-IFR 88 ; CNRS-Universités Aix Marseille II et III ; BP 71; 31 Chemin Joseph Aiguier; 13402 Marseille Cedex 20; France; ${ }^{\dagger}$ The Pasarow Mass Spectrometry Laboratory; NPI - Semel Institute for Neuroscience and Human Behavior; University of California; Box 42, NPI-Semel Institute Room \# 68146, 760 Westwood Plaza; Los Angeles; California; 90024-1759; USA.

Short title: Mapping the $\mathrm{CP} 12 \mathrm{Cu}^{2+}$ binding site

Address for correspondence to (present address): $\operatorname{Dr}$ Frédéric Halgand. Laboratoire de physiologie de la reproduction; Unité Mixte de Recherche INRA - CNRS (UMR 6175), F-37380 Nouzilly (France) ; Université François Rabelais de Tours, F-37380 Nouzilly (France) ; Haras nationaux, F-37380 Nouzilly; France. Phone: (33) 2474279 49; Fax: (33) 247427743. Email: Frederic.Halgand@tours.inra.fr. 


\section{SYNOPSIS:}

CP12 is a small chloroplastic protein involved in the Calvin cycle that was shown to bind copper, a metal ion involved in modulation of its transition from reduced to oxidized state. In order to describe CP12 copper binding properties, copper-IMAC experiments and site-directed mutagenesis based on computational modeling, were coupled to top-down mass spectrometry (ESI-MS and MSMS). Copper-IMAC experiments allowed the primary characterization of mutation effects upon copper binding. Top-down MSMS experiments carried out under nondenaturing conditions on wild-type and mutant $\mathrm{CP} 12 / \mathrm{Cu}^{2+}$ complexes then allowed fragment ions specifically liganding the copper ion to be determined. Comparison of MSMS datasets defined three regions involved in metal ion binding: residues D16 to D23, D38 to K50 and D70 to E76, with the two first regions containing selected residues for mutation. These data confirmed that copper ligands involved glutamic and aspartic residues in contrast to typical protein copper chelators. We propose that copper might play a role in regulation of CP12 biological activity. (160 words).

KEYWORDS: CP12; top down; mass spectrometry; Calvin cycle; copper; redox transition.

1 Abbreviations used are: $\mathrm{CD}=$ Circular Dichroism; $\mathrm{CS}=$ Charge State; $\mathrm{CSD}=$ Charge State Distribution; $\mathrm{CID}=$ Collisionally Induced Dissociation; $\mathrm{CAD}=$ Collisionally Activated Dissociation; ESI = Electrospray Ionization; FTICR-MS = Fourier Transform Ion Cyclotron resonance mass spectrometry; GAPDH = Glyceraldehyde-3-Phosphate Dehydrogenase; HCD = Higher energy Collisional Dissociation; IMAC $=$ Immobilized Metal Ion Affinity Chromatography; MALDI-MS = Matrix Assisted Laser Desorption ionization mass spectrometry; MS = Mass Spectrometry; MSMS = Tandem mass spectrometry; PQD = Pulsed-Q Dissociation; PRK = Phosphoribulokinase; RMS = Root Mean Square; Zav = mean charge state; $\sigma(i)=$ standard error on Zav value 


\section{INTRODUCTION:}

CP12 is a small chloroplastic protein of about 80 residues that is involved in the Calvin cycle responsible for $\mathrm{CO}_{2}$ assimilation in photosynthetic organisms. It has been found in higher plants [1], green [2] and red algae [3], in the cyanobacterium Synechocystis PCC6803 [2] and recently in a freshwater diatom, Asterionella formosa $[4,5]$.

$\mathrm{CP} 12$, in higher plants and green algae, has four conserved cysteine residues that are believed to be functionally important and shares sequence similarities with a 21 amino-acid long Cterminal sequence extension of the glyceraldehyde-3-phosphate dehydrogenase (GAPDH) B subunit that contains the regulatory cysteine residues [6]. CP12 is involved in the regulation of GAPDHs that lack this C-terminal extension such as $\mathrm{A}_{4}$ GAPDH from cyanobacteria [7] and green algae [8-10]. CP12 forms a protein complex with phosphoribulokinase (PRK) and GAPDH. Putative N- and C- terminal peptide loops formed via disulfide bonds are involved in the interaction with PRK and GAPDH, respectively $[1,2]$. The role of CP12 in the assembly process of the PRK/GAPDH/CP12 complex in Chlamydomonas reinhardtii [11, 12] and in Arabidopsis thaliana $[13,14]$ has been extensively characterized. However, CP12 belongs to the intrinsically unstructured protein (IUP) family, members of which are involved in protein-protein interactions that often play major roles in cellular functions [15]. This suggests that CP12 could be involved in protein recruiting in other metabolic pathways. Such a feature was recently reported with the demonstration that $\mathrm{CP} 12$ can bind to aldolase [16]. In addition, CP12 was found to bind a metal ion [17] in C. reinhardtii and using non-covalent ESI-MS experiments, the oxidized protein was found to bind $\mathrm{Cu}^{2+}$ specifically ( $\mathrm{Kd}$ of $26 \pm 1 \mu \mathrm{M}$ ). It was also shown that $\mathrm{Cu}^{2+}$ catalyzes the reformation of the disulfide bonds of the reduced CP12, leading to the recovery of fully oxidized CP12. This study also showed that CP12 has a high structural similarity with copper chaperones from $A$. thaliana, proteins that play different roles in copper homeostasis [18-20]. The copper ion binding site on CP12 was not identified but clearly does not involve cysteine residues.

Top-down mass spectrometry is an emerging tool for obtaining structural information from intact proteins by fragmenting them by tandem mass spectrometry [21, 22]. The use of top-down MS in proteomics has led to several studies reporting the characterization of new protein variants, isoforms and post-translational modifications [23-25]. Such methodology was also used for the discovery of biomarkers [26] and was shown to be promising for the analysis of membrane proteins [27-29]. Non-covalent protein assemblies were also shown to be accessible by top-down MSMS experiments [30, 31]. The impact of this new field of investigation is illustrated with the discovery of more extensive polymorphisms and structural complexity to proteins than previously envisaged [32-34].

In this study, we used top-down mass spectrometry coupled with site-directed mutagenesis and biochemical analysis to map the copper binding site of C. reinhardtii CP12.

\section{EXPERIMENTAL:}

\section{Materials}

$\mathrm{CdSO}_{4}, \mathrm{CuCl}_{2}, \mathrm{FeCl}_{2}$ were supplied by Merck. Ammonium bicarbonate was purchased from Sigma.

Mutation and purification of recombinant CP12. Based on previous MS experiments showing the removal of two protons upon copper fixation to $\mathrm{CP} 12$ [17] and on sequence 
alignment of CP12 from various species [3, 35] site-directed mutageneses were performed. The codon (GAG) for glutamate residues (E20, E41 and E42) in the Chlamydomonas CP12 coding sequence, in plasmid pGEMT (Promega, Madison, USA), was mutated to alanine (GCG) or lysine (AAG) using the QuickChange ${ }^{\mathrm{TM}}$ site-directed mutagenesis kit (Stratagene, La Jolla, CA USA) and their incorporation confirmed by Big-Dye DNA sequencing and ESI-MS (see below). Wild-type and mutant CP12 was expressed in Escherichia coli using a pET16b plasmid and purified by IMAC as described [11]. The N-terminal histidine tag sequence (HHHHHHHHHHSSGHIEGR) removed using Factor Xa (Merck KGaA, Darmstadt, Germany) leaving an additional His-1 and Met-2 appended to the native CP12 N-terminus (SGQPA; swissprot acc. A6Q0K5). CP12 sequences were numbered with these two additional residues.

Circular Dichroism Studies on CP12 proteins. Spectra were recorded on a Jasco J-815 CD spectrometer (JASCO, Bouguenais, France). All spectra are averages of two accumulations from 260 to $190 \mathrm{~nm}$ with a $10 \mathrm{~nm} / \mathrm{min}$ scan rate at $23^{\circ} \mathrm{C}$. Prior to $\mathrm{CD}$ experiments $\mathrm{CP} 12$, without the histidine tag, was dialyzed against phosphate buffer (10 mM Na $2 \mathrm{HPO}_{4}-\mathrm{NaH}_{2} \mathrm{PO}_{4}, \mathrm{pH}$ 6.0). Measurements were performed using a $0.2 \mathrm{~cm}$ quartz vials in a final volume of $350 \mu \mathrm{L}$ and for a $10 \mu \mathrm{M}$ concentration of CP12 proteins. For each sample, the fractional $\alpha$-helical content was calculated using the assumption that for $100 \% \alpha$-helix the mean residue ellipticity [ $\theta$ ] at $222 \mathrm{~nm}$ is $[\theta]_{222}=-36,300(1-2.57 / \mathrm{X})$, where $\mathrm{X}$ is the number of residues in the protein [36].

Fluorescence studies. Fluorescence spectra of CP12 proteins were recorded on a Fluorolog-3 Jobin Yvon-Spex spectrometer (HORIBA Jobin Yvon SAS, Longjumeau, France) and were recorded from 300 to $450 \mathrm{~nm}$ with an excitation wavelength of $290 \mathrm{nM}$ at $23{ }^{\circ} \mathrm{C}$. Measurements were performed with a scan speed of $1 \mathrm{~nm}$ per $2 \mathrm{sec}$. CP12 proteins were diluted to $1.8 \mu \mathrm{M}$ in 50 $\mathrm{mM}$ Tris- $\mathrm{HCl}, 100 \mathrm{mM} \mathrm{NaCl}$ at $\mathrm{pH} 8$ in a $1 \mathrm{~cm}$ quartz vials.

CU-IMAC and dot blot analyses of copper binding properties of wild-type CP12 and mutants. IMAC columns (ZipTip ${ }_{\mathrm{MC}}$, Millipore Corp., Billerica, MA USA) were first loaded with copper using a $200 \mathrm{mM} \mathrm{CuSO}_{4}$ solution except for the control experiment where copper was replaced by $200 \mathrm{mM} \mathrm{FeCl}_{2}$. All steps were done according to the manufacturer protocol. CP12 proteins were loaded on the Cu-IMAC column at $0.1 \mathrm{mg} / \mathrm{ml}$. Screening of the differential protein behaviors upon copper binding were revealed using dot blot experiments performed on each washing and elution step. Strong metal binding affinity was systematically assessed by adding an EDTA elution step. Aliquot samples of the different eluted fractions of the IMAC column were spotted were spotted onto nitrocellulose membranes (Minifold I, dot/slot system SRC 96, Schleicher and Schüll, Dassel, Germany). The nitrocellulose filters were then probed with a rabbit antiserum directed against recombinant $C$. reinhardtii CP12 (1/3000). Antibody binding was revealed using Enhanced Chemiluminescence (ECL) detection system (GE Healthcare, Uppsala, Sweden) using anti-rabbit IgG / horseradish peroxidase-linked antibody $(1 / 10000)$ from donkey.

Sample preparation for mass spectrometry. Prior to mass spectrometry (MS) experiments, CP12 was desalted using an ultra-free membrane filter with a "cut-off" of 3,000 Da (Millipore Corporation, Bedford, MA USA). CP12 concentration was then determined with the Bradford assay using bovine serum albumin (BSA) as standard [37]. For measurements under nondenaturing conditions, the protein was diluted to $5 \mathrm{uM}$ in $25 \mathrm{mM}$ ammonium bicarbonate, $\mathrm{pH}$ 6.8. Copper and cadmium were added at a $1 / 5$ fixed protein/metal ratio. His tag was removed in order 
to avoid unspecific metal binding and to improve the fragmentation yield in MSMS for maximal coverage of the sequence.

Electrospray mass spectrometry (ESI-MS). All samples were analyzed using a LTQOrbitrap hybrid mass spectrometer (Thermo-Fisher Company, Bremen, Germany) fitted with a nanospray ion source. MS and MSMS spectra were recorded in the FTICR-MS mode, at 60000 resolution using normal scan rate mode at 3 micro scans and a maximum injection time of 200 ms. Instrumental parameters were optimized in order to obtain the best sensitivity without disrupting non-covalent interactions (positive ion mode, centroid format, ISV $=1.3 \mathrm{kV}$, ddp $=$ $150 \mathrm{~V}$, temperature of the transfer capillary $=150{ }^{\circ} \mathrm{C}$ ). Mass spectrometer was calibrated in the $500-2000 \mathrm{~m} / \mathrm{z}$ range using the standard Ultramark ${ }^{\mathrm{TM}}$ solution provided by manufacturer (Thermo-Fisher Company, Bremen, Germany). RMS calculated on MSMS fragments were typically below $2 \mathrm{ppm}$. When necessary, the $\mathrm{m} / \mathrm{z}$ range was extended to 4000 without recalibrating the instrument. In that case the mass accuracy decreased a little with a RMS below 5 ppm. All available collisional modes such as CID, CAD, PQD and HCD were tested. It appeared that standard CAD mode gave the best results and was finally used for all MSMS experiments. For each sample, MSMS experiments were performed on the main charge state $(5+)$ and for the same deposited collisional energy $(20 \mathrm{eV})$. When necessary other charge states were fragmented to increase sequence coverage. All MSMS spectra were recorded in the averaging mode to improve the signal-to-noise ratio. Reproducibility of these experiments was assessed. Prosight PTM software developed by Neil Kelleher's group was used (https://prosightptm.scs.uiuc.edu) to data mined MSMS data with a 5 ppm mass accuracy. Results gave excellent "Crude Pscores" assessing protein sequence identities with RMS of fragment masses between 0.97 and $1.5 \mathrm{ppm}$.

\section{RESULTS}

CP12 mutagenesis. Copper binding to $\mathrm{CP} 12$ promotes the removal of two protons and the conserved His-49 is not involved in this binding [17], it was thus speculated that acidic residues may be involved in $\mathrm{Cu}^{2+}$ binding. From an alignment of $\mathrm{CP} 12$ from various species $[3,34]$ three conserved Glu residues (E20, E41 and E42) were identified as good candidates to bind copper ions (Figure 1). Consequently, these residues in C. reinhardtii $\mathrm{CP} 12$ were mutated to either alanine or lysine. Eight CP12 mutants were examined: E20A, E41A, E42A, E41A/E42A, E20K, $\mathrm{E} 41 \mathrm{~K}, \mathrm{E} 42 \mathrm{~K}$ andE41K/E42K. MS analysis of the purified wild-type and mutant CP12s showed that their experimental monoisotopic molecular weights matched their expected masses (Supplementary material Table 1A). Top-down MSMS data were recorded for each free CP12 protein. All MSMS data acquired for the wild-type CP12 and mutants unambiguously confirmed the expected sequences and location of mutations. CP12 protein sequences and corresponding $\mathrm{b}$ and y fragment ions for the free species are displayed in Figure 1, supplementary material.

Circular Dichroism spectroscopy and fluorescence. The effect of the glutamate mutations on secondary structure was analyzed by circular dichroism (CD) spectroscopy (Figure 2A). The similarities in the CD spectra for the wild-type and single mutant CP12s indicated that they shared similar secondary structure with only slight reductions in the alpha-helical content. This content was about $25 \%$ for E20A, $26 \%$ for E41A and $23 \%$ for E42A while it is about $29 \%$ for the wild-type. In contrast, the decrease in the alpha-helix content in E41A/E42A (17\%) and $\mathrm{E} 41 \mathrm{~K} / \mathrm{E} 42 \mathrm{~K}(19 \%)$ mutants was more prominent indicating a more substantial change in the global structure of these double mutants. The influence of $\mathrm{Cu}^{2+}$ on the $\mathrm{CD}$ spectra of wild-type 
and mutant CP12s was also examined and showed that only the wild-type CP12 spectra was influenced by copper in which the alpha-helix content slightly increased to $32 \%$ (Figure $2 \mathrm{~A}$ ). No effect of copper was observed on each mutant (Table 1).

Fluorescence spectra obtained with wild-type and mutant CP12s revealed no difference between E42A mutant and wild-type CP12 whereas a quenching of tryptophan fluorescence was observed for all other mutants (Figure 2B). Result obtained for E42A mutant was consistent with the fact that no hydrophobic contact could be contracted with this amino acid since no apolar residue is in its vicinity. Quenching effects observed for other mutants were quantified and a scale was defined according to the mutation effect upon structure, with the following order $\mathrm{E} 41 \mathrm{~K} / \mathrm{E} 42 \mathrm{~K}>\mathrm{E} 20 \mathrm{~A}$ and E41A/E42A $>$ E41A > wild-type.

Mutation effects on copper binding. The copper chelation properties of wild-type CP12 and mutants were tested using IMAC resin charged with $\mathrm{Cu}^{2+}$ (IMAC-Cu). As wild-type CP12 can bind copper, it bound to IMAC-Cu and can only be eluted by $0.3 \mathrm{M} \mathrm{NH} 4 \mathrm{OH}$ or $1 \mathrm{mM}$ EDTA. In contrast, mutants with impaired affinities for copper did not bind to IMAC-Cu or eluted during the washing steps. The affinity of the mutant CP12s was qualitatively assessed by monitoring the eluent for CP12 by immunodetection (Table 2). Based on their affinity to IMAC-Cu, mutation of E20 had no influence on $\mathrm{Cu}$ binding while the mutation of E41 strongly affected copper binding and that of E42 into $\mathrm{K}$ only diminished copper binding. Wild-type CP12 did not bind to IMAC resin charged with $\mathrm{Fe}^{2+}$ indicating that the binding of CP12 to IMAC-Cu was copper specific.

Assessment of copper binding. Wild-type CP12 and mutants molecular masses were experimentally measured after adding a $1 / 5$ protein/copper ratio. In each case, mass spectra only displayed the presence of one copper atom complexed to CP12. Calculated molecular masses showed that copper binds to all CP12 proteins (wild-type and mutants) with a mean monoisotopic mass increment of $60.91 \mathrm{Da}$ (see Table 1B, supplementary material). This confirms previous observations that copper redox state binding is $2+[38,39]$ and tetragonal coordination of $\mathrm{Cu}^{2+}$ is therefore proposed in accordance with [40]. The chemical formula describing the $\mathrm{CP} 12-\mathrm{Cu}$ complex is $[\mathrm{M}-2 \mathrm{H}+\mathrm{Cu}+\mathrm{nH}]^{\mathrm{n}+}$ where $\mathrm{M}$ is the mass of the protein with subtraction of the mass of two hydrogens linked to copper co-ordination, plus addition of the mass of copper (explaining the mass increment of 60.91 observed) and n, protons giving the charge state. Following this statement the main charge state of the wild-type CP12 protein complexed with copper being can be expressed as followed: [8729.0587 - $(2 \times 1.0078)+62.9296$ $+(5 \times 1.0078)]^{5+}$ with an expected monoisotopic $\mathrm{m} / \mathrm{z}$ value at 1759.0023 in agreement with experimental value observed at 1759.0054 (1.73 ppm difference). Deconvolution software was found to give the correct mass but peak charge states were manually checked and found in agreement with automated charge state assignments. A noticeable difference in the capacity of the $\mathrm{E} 41 \mathrm{~K} / \mathrm{E} 42 \mathrm{~K}$ CP12 variant to bind copper ion was observed. While the wild-type and other mutant $\mathrm{CP} 12$ s fully bound $\mathrm{Cu}^{2+}$ within $2 \mathrm{~min}$, incubation for $24 \mathrm{~min}$ was required to bind copper to $\sim 50 \%$ of the E41K/E42K CP12 mutant. The reduced binding affinity might be attributed to a higher steric hindrance imparted by the length and protonation propensity of the Lys side chains (Figure 3).

Studying protein CSD. Since it was demonstrated that variations of protein charge state distribution (CSD) could be related to structural properties, we used that feature to probe conformational changes linked to mutations and copper binding. In particular, we used Zav and $\sigma(i)$ values that correspond to the calculated mean charge state and standard deviation respectively [41]. Calculated Zav values and calculated standard errors [42] describing mutation 
effects and copper binding influence on structure are reported in Table 3. Results of CSD analysis display no significant change between wild-type CP12 and mutants except for E41K/E42K double mutant that presents a slight effect. Similarly, comparison of data obtained for the free and copper complexed states of each protein, did not reveal important change. The slight difference observed for E20A and E42A mutants being in these cases assigned to a gas phase effect related to the strengthening of electrostatic bonds in a lower dielectric environment (vacuum).

MSMS of $\mathrm{Cu}^{2+} / \mathrm{CP12}$ complexes. Our approach was validated by recording MSMS data for the $\mathrm{CP} 12 / \mathrm{Cd}^{2+}$ complex (control experiment), a metal ion that was shown to bind un-specifically to CP12 [17] and in that case, cadmium was observed as adduct on every fragment ion. Intensities of fragment ions bearing a $\mathrm{Cd}^{2+}$ adduct were minor and in mixture with the corresponding free fragment ions. In contrast, MSMS recorded for $\mathrm{CP} 12 / \mathrm{Cu}^{2+}$ complexes showed the presence of specific fragment ions bearing one copper ion, representing in that case the major species.

Extractions of MSMS data were realized for spectra having the same acquisition time. The detection of artifactual fragment peaks with Prosight PTM was observed, due to missassignments of peaks and/or isotopic profiles. Therefore, some peaks were manually removed from the peak lists and charts to clarify the figures. Automated searches also led to the "recognition" of peaks assigned to fragment ions located between the two cysteine residues linked through a disulfide bridge. Since the breakage of disulfide links is un-expected under low collisionally energy mode or required mechanisms involving charge directed neighboring group or non mobile proton salt processes [43], we manually checked the presence of these ions. Scrutiny of MSMS spectra finally proved the presence of such ions (b70 ions, labeled with an asterisk in sequence charts).

A couple of MSMS spectra are shown in Figure 4. Less fragment ions were observed on MSMS spectra recorded for the $\mathrm{CP} 12 / \mathrm{Cu}^{2+}$ complexes compared to their respective free states (Figure 5A). This suggests that binding of copper triggered conformational changes leading to a more rigid structure. However, the yield of fragmentation differed from one protein to another, with E41A mutant showing the strongest "stabilizing" effect linked to copper binding.

Copper was not detected on fragment ions located on $\alpha$-helix two (residues 30 to 56) until position 52 is reached (fragment b52), probably because crucial interactions between the two helices are kept. Similarly, the proximity of the disulfide bridges seemed to be essential to maintain a minimal structure allowing the ligation of a copper ion (ex: y20 fragment ion). Three regions putatively involved in copper binding were proposed. Regions 1 to 3 spanned from residues D16 to D23 (fragments b16 to b23), residues D38 to K50 (fragments b38 to b50) and residues D70 to E76 (fragments b70 to b76), respectively (see locations in Figure 5B). Defining the first region (D16 to D23 in regular nomenclature) including the E20 residue was straight forward, with the recurrent detection of b16, b22 and b23 ions bearing a copper ion for all CP12 species. Location of the second region (D38 to K50) comes from the detection of overlapping b (b52 to b62) and y (y25 to y44) fragment ions bearing a copper ion. We narrowed region 2 to residues 38 to 50 because CP12 secondary structure upholding strongly depends on interactions between residues 11 to 16 of the N-terminus and residues 53 to 58 of the second $\alpha$-helix [44]. Identification of the third putative region was based on the recurrent detection of y 20 and y 25 fragment ions as well as b70 ion observed for E20A/ $\mathrm{Cu}^{2+}$ and $\mathrm{E} 42 \mathrm{~A} / \mathrm{Cu}^{2+}$ complexes MSMS spectra. As mentioned above, since we proved the real presence of b70 ions in MSMS spectra 
and that disulfide breakage can only occur via a mechanism involving a fixed proton [43], observation of b70 ions can only be explained by the presence of $\mathrm{Cu}^{2+}$ in a close proximity of the C68-C77 disulfide bridge.

A detailed description is proposed to explain the detection of the b70 ions only for E20A and E42A MSMS spectra. For E20A, mutation is proposed to promote a shift of the first $\alpha$-helix that became closer to $\alpha$-helix two due to the smaller steric hindrance of the side chain. Bringing the two helices together can induce a movement of the copper ion toward the C68-C77 disulfide bridge then inducing its breakage. This explanation is in concordance with the fluorescence data showing that E20A mutation induces an important quench of Trp 37 suggesting rearrangements of the two $\alpha$-helices. In the second case, mutant E42A is proposed to induce disulfide bridge reduction in a similar manner by bringing together the C-terminal loop and $\alpha$-helix two, by reinforcing hydrophobic contacts with Phe 67, for example. This explanation could be in apparent discrepancy with fluorescence data showing no change in Trp37 fluorescent spectrum. However, the presence of a unique Trp in CP12 sequence allows us to monitor structural changes only in its vicinity. The absence of b70 ion in MSMS spectra of E41A mutant complexed with copper could be explained by a strengthening of the hydrophobic contacts of the two $\alpha$-helices. In that case copper ion would not be directed toward the C-terminal disulfide bridge and no disulfide bond breakage will occur. Finally the lack of $b 70$ ion on MSMS spectra recorded for double mutants could be explained by any structural change pushing the copper ion remotely from the C-terminal disulfide bridge. All these results support the presence of copper in the Cterminal part of the protein. Finally, the model structure of $\mathrm{CP} 12$ protein shows that the loop made by the C-terminal disulfide bond contains four acidic residues (D70, D73 and D75, E76), in close vicinity to residues D38 and E42, and thus may be involved in copper co-ordination. Region 3 was proposed to span from residues D70 to E76. Experimental values of fragments carrying a copper ion compared to theoretical ones are given in Tables 2 to 7 of supplementary material.

\section{DISCUSSION}

Although CP12 can bind copper ion [17], no information on the location of the binding site of this metal ion has been available so far thereby impeding progress in understanding its function. After selecting residues presumably involved in copper binding, global structure of the mutants was checked using circular dichroism (CD), fluorescence as well as MS. Circular dichroism showed that global structure of single mutants was not affected by mutations whereas double mutants were shown to have a higher impact on CP12 tertiary and secondary structure. Fluorescence data indicated quenching effects of Trp 37 for E20A, E41A, E41A/E42A and $\mathrm{E} 41 \mathrm{~K} / \mathrm{E} 42 \mathrm{~K}$ mutants, in agreement with $\mathrm{CD}$ experiments also demonstrating that single mutations had little influence (not for E20A) on CP12 structure whereas double mutants were shown to significantly alter the CP12 protein conformation (Figure 2). Analysis of Zav values deduced from charge state distribution (CSD), and differences of these values between mutants and wild-type was then used to confirm structural changes observed with $\mathrm{CD}$ and fluorescence experiments. Unfortunately, these values did not allowed characterization of structural effects of mutations or the impact of copper binding upon CP12 structure. The global protonatable surfaces of mutants are similar to wild-type CP12 and the extent of structural changes linked to copper binding does not affect the global structure of the protein. 
The effects of the Glu mutations on copper binding by CP12 were also examined using IMAC-Cu which indicated E41 has a strong influence on copper binding (Table 2). Mutation of E20 had little or no influence on the avidity of CP12 for $\mathrm{Cu}^{2+}$ while the substitution of E42 with Lys impeded copper binding, possibly due to a reverse charge effect or steric hindrance. An interesting feature of the IMAC-Cu binding assay is the likely requirement for the residues involved in the co-ordination with the immobilized $\mathrm{Cu}^{2+}$ to be appropriately exposed to the solvent (i.e. arranged on the protein surface). This may explain why the E41A mutant was shown by MS to bind copper while having reduced affinity for IMAC-Cu.

To better grasp the structure-function relationship of CP12 copper binding properties, topdown MSMS experiments were performed on free and $\mathrm{Cu}$-complexed states of each CP12 protein. Top-down MSMS experiments not only allowed the protein sequence of the wild-type and mutants to be assessed but also confirmed the location of the disulfide bridges.

ESI and particularly nanoESI ionization techniques produce ions with minimal ion fragmentations and internal rearrangements $[38,45]$. Based on the fact that no change was observed in Zav values, mutations seem not to affect the global desorption-ionization efficiency of CP12 mutants compared to wild-type. Since we used identical MSMS parameters and since control experiments conducted with $\mathrm{Cd}^{2+}$ confirmed that detection of fragment ions carrying a copper ion are significant, results deduced from top-down MSMS were shown to directly reflect copper binding effects in solution. This study further supports the involvement of D and E residues in metal ion co-ordination with the location of three regions (region $1=\mathrm{D} 16$ to D23; region $2=\mathrm{D} 38$ to $\mathrm{K} 50$ and region $3=\mathrm{D} 70$ to $\mathrm{E} 76$ ) putatively involved in copper binding. These results are summarized in Figure 5B. Detection of b70 ions that were only found for E20A and E42A mutants $/ \mathrm{Cu}^{2+}$ complexes, demonstrates the proximity of the copper ion binding to the $\mathrm{C}$ terminal disulfide bond. Such fragmentation, according to the literature, could only be related to mechanisms involving charge directed neighboring group or non mobile proton salt processes [43]. These mechanisms were confirmed by the detection of specific chemical losses (-44 Da and $-45 \mathrm{Da}$ ) from b or y fragment ions bearing a copper ion [43].

MSMS data suggest that there is a relationship between the structural changes induced by the mutations and the impairment of copper binding. Types of modifications involved in these structural changes include rupture of electrostatic network or enhancement of hydrophobic interactions. This is illustrated with E20A and E41A mutants that were shown to induce structural changes in the vicinity of Trp37 where stabilization of the hydrophobic core of the protein between the two $\alpha$-helices is proposed. Scrutiny of the predicted CP12 structure showed that the two helices are maintained by a hydrophobic core, in concordance with the stabilization promoted by E41A mutation. This could also be the case for E42A mutant, but with a more moderated effect on structure. In that case, the rupture of electrostatic network involved in copper binding with the C-terminal loop is proposed to be compensated by reinforcement of hydrophobic contacts with Phe67. This suggestion is supported by description of strong interactions between Phe67 and Ala45 and 46 [44].

At this stage, one intriguing point remained to be explained. If regions 2 and 3 are relatively close in CP12 structure allowing copper chelation, region 1 is however remotely located on the CP12 structure. The detection of b16 to b23 fragment ions bearing a copper ion means that any residue between positions 1 to 23 can fix the copper ion. Moreover, no candidate was found in the close vicinity of this region to allow copper binding. To bridge the gap between all our observations, we proposed that carbonyl groups of the $\alpha$-helix peptidic backbone could be 
involved in the binding of the metal ion. Such a hypothesis can link the hydrophobic and electrostatic effects observed. Moreover, the implication of one of the acidic residues present inside the C-terminal disulfide loop, such as D70 or D73 or even the C-terminal carboxylate, in tetragonal co-ordination of copper is not ruled out.

The C-terminal domain of the $\mathrm{CP} 12$ protein plays a major role in protein-protein interactions $[1,46]$. GAPDH and PRK interact with CP12 even in the absence of copper, thus, binding of copper to CP12 is not necessary for the formation of the GAPDH/PRK/CP12 complex. Dissociation of this complex in vivo is triggered by a light dependent ferredoxin-thioredoxin system and linked to the reduction of the two disulfide bridges of the CP12 protein $[8,14,46]$. In contrast, under dark conditions where formation of this complex occurs, formation of disulfide bridges on CP12 could be triggered either by oxidized thioredoxin or by copper binding, thus leading to oxidized CP12. However, the role of the binding of a copper ion on the three regions defined in this report still remains to be elucidated but could be linked to a specific feature of CP12. Indeed, this protein belongs to the intrinsically unstructured protein (IUP) family [11] and a multitude of examples has demonstrated that the function of disordered proteins can be closely related to their metal ion binding properties. To cite but a few examples: the prion protein can bind copper ion and as a consequence plays a role in amyloid formation [47], the $\mathrm{N}$-terminal domain of HIV integrase can bind $\mathrm{Zn}^{2+}$ leading to an increased activity [48], and the $\alpha$-synuclein can bind many metal ions thereby accelerating its fibrillation [49]. We showed here that binding of copper triggered conformational changes on $\mathrm{CP} 12$ leading to a more rigid structure. Reduction in plasticity and/or conformational changes upon metal ion binding thus might represent a general mechanism for enhancing the target recognition of IUP and in particular, of CP12.

\section{Acknowledgments}

Jenny Erales is grateful to the French Ministry of Research for $\mathrm{PhD}$ funding (fellowship from the Université Pierre et Marie Curie, Paris VI, France). Authors are grateful to Dr Jean-Yves Salpin and Dr Aram Nersissian for helpful scientific discussion.

\section{REFERENCES}

1 Wedel, N., Soll, J. and Paap, B. K. (1997) CP12 provides a new mode of light regulation of Calvin cycle activity in higher plants. Proc. Natl. Acad. Sci. U S A. 94, 10479-10484.

2 Wedel, N. and Soll, J. (1998) Evolutionary conserved light regulation of Calvin cycle activity by NADPH-mediated reversible phosphoribulokinase/CP12/ glyceraldehyde-3phosphate dehydrogenase complex dissociation. Proc. Natl. Acad. Sci. U S A. 95, 9699-9704.

3 Oesterhelt, C., Klocke, S., Holtgrefe, S., Linke, V., Weber, A. P. and Scheibe, R. (2007) Redox regulation of chloroplast enzymes in Galdieria sulphuraria in view of eukaryotic evolution. Plant Cell. Physiol. 48, 1359-1373.

4 Boggetto, N., Gontero, B. \& Maberly, S. C. (2007) Regulation of phosphoribulokinase and glyceraldehyde 3-phosphate dehydrogenase in a freshwater diatom, Asterionella formosa, (Bacillariophyceae). J. Phycol. 43, 1227-1234.

$5 \quad$ Erales, J., Maberly, S \& Gontero, B. (2008) Specificity and function of glyceraldehyde 3phosphate dehydrogenase in a freshwater diatom, Asterionella formosa (Bacillariophyceae). J. Phycol. 44, 1455-1464. 
6 Pohlmeyer, K., Paap, B. K., Soll, J. and Wedel, N. (1996) CP12: a small nuclear-encoded chloroplast protein provides novel insights into higher-plant GAPDH evolution. Plant Mol. Biol. 32, 969-978.

7 Tamoi, M., Miyazaki, T., Fukamizo, T. and Shigeoka, S. (2005) The Calvin cycle in cyanobacteria is regulated by $\mathrm{CP} 12$ via the $\mathrm{NAD}(\mathrm{H}) / \mathrm{NADP}(\mathrm{H})$ ratio under light/dark conditions. Plant J. 42, 504-513.

8 Lebreton, S., Andreescu, S., Graciet, E. and Gontero, B. (2006) Mapping of the interaction site of CP12 with glyceraldehyde-3-phosphate dehydrogenase from Chlamydomonas reinhardtii. Functional consequences for glyceraldehyde-3-phosphate dehydrogenase. Febs J. 273, 3358-3369.

9 Graciet, E., Mulliert, G., Lebreton, S. and Gontero, B. (2004) Involvement of two positively charged residues of Chlamydomonas reinhardtii glyceraldehyde-3-phosphate dehydrogenase in the assembly process of a bi-enzyme complex involved in $\mathrm{CO}_{2}$ assimilation. Eur. J. Biochem. 271, 4737-4744.

10 Graciet, E., Lebreton, S. and Gontero, B. (2004) Emergence of new regulatory mechanisms in the Benson-Calvin pathway via protein-protein interactions: a glyceraldehyde-3phosphate dehydrogenase/CP12/phosphoribulokinase complex. J. Exp. Bot. 55, 1245-1254.

11 Graciet, E., Gans, P., Wedel, N., Lebreton, S., Camadro, J. M. and Gontero, B. (2003) The small protein CP12: a protein linker for supramolecular assembly. Biochemistry. 42, 81638170 .

12 Graciet, E., Lebreton, S., Camadro, J. M. and Gontero, B. (2003) Characterization of native and recombinant A4 glyceraldehyde 3-phosphate dehydrogenase. Kinetic evidence for confromation changes upon association with the small protein CP12. Eur. J. Biochem. 270, 129136.

13 Marri, L., Trost, P., Pupillo, P. and Sparla, F. (2005) Reconstitution and properties of the recombinant glyceraldehyde-3-phosphate dehydrogenase / CP12 / phosphoribulokinase supramolecular complex of Arabidopsis. Plant Physiol. 139, 1433-1443.

14 Marri, L., Trost, P., Trivelli, X., Gonnelli, L., Pupillo, P. and Sparla, F. (2008) Spontaneous Assembly of Photosynthetic Supramolecular Complexes as Mediated by the Intrinsically Unstructured Protein CP12. J. Biol. Chem. 283, 1831-1838.

15 Uversky, V. N. and Fink, A. L. (2004) Conformational constraints for amyloid fibrillation: the importance of being unfolded. Biochim. Biophys. Acta. 1698, 131-153.

16 Erales, J., Avilan, L., Lebreton, S. and Gontero, B. (2008) Exploring CP12 binding proteins revealed aldolase as a new partner for the phosphoribulokinase/glyceraldehyde 3phosphate dehydrogenase/CP12 complex--purification and kinetic characterization of this enzyme from Chlamydomonas reinhardtii. Febs J. 275, 1248-1259.

17 Delobel, A., Graciet, E., Andreescu, S., Gontero, B., Halgand, F. and Laprevote, O. (2005) Mass spectrometric analysis of the interactions between CP12, a chloroplast protein, and metal ions: a possible regulatory role within a PRK/GAPDH/CP12 complex. Rapid. Commun. Mass Spectrom. 19, 3379-3388.

18 Himelblau, E., Mira, H., Lin, S. J., Culotta, V. C., Penarrubia, L. and Amasino, R. M. (1998) Identification of a functional homolog of the yeast copper homeostasis gene ATX1 from Arabidopsis. Plant Physiol. 117, 1227-1234.

19 Mira, H., Martinez-Garcia, F. and Penarrubia, L. (2001) Evidence for the plant-specific intercellular transport of the Arabidopsis copper chaperone CCH. Plant J. 25, 521-528. 
20 Mira, H., Vilar, M., Perez-Paya, E. and Penarrubia, L. (2001) Functional and conformational properties of the exclusive C-domain from the Arabidopsis copper chaperone (CCH). Biochem. J. 357, 545-549.

21 Kelleher N. L., Lin H. Y., Valaskovic G. A., Aaserud D. J., Fridriksson E. K. and McLafferty F. W. (1999) Top down versus bottom up protein characterization by tandem highresolution mass spectrometry. J. Am. Chem. Soc. 121, 806-807.

22 Forbes, A. J., Patrie, S. M., Taylor, G. K., Kim, Y. B., Jiang, L. and Kelleher, N. L. (2004) Targeted analysis and discovery of posttranslational modifications in proteins from methanogenic archaea by top-down MS. Proc. Natl. Acad. Sci. U S A. 101, 2678-2683.

23 Deterding, L. J., Bhattacharjee, S., Ramirez, D. C., Mason, R. P. and Tomer, K. B. (2007) Top-Down and Bottom-Up Mass Spectrometric Characterization of Human Myoglobin-Centered Free Radicals Induced by Oxidative Damage. Anal. Chem. 79, 6236-6248.

24 Patrie, S. M., Ferguson, J. T., Robinson, D. E., Whipple, D., Rother, M., Metcalf, W. W. and Kelleher, N. L. (2006) Top down mass spectrometry of $<60-\mathrm{kDa}$ proteins from Methanosarcina acetivorans using quadrupole FRMS with automated octopole collisionally activated dissociation. Mol. Cell. Proteomics. 5, 14-25.

25 Pesavento, J. J., Garcia, B. A., Streeky, J. A., Kelleher, N. L. and Mizzen, C. A. (2007) Mild performic acid oxidation enhances chromatographic and top down mass spectrometric analyses of histones. Mol. Cell. Proteomics. 6, 1510-1526.

26 Pisitkun, T., Johnstone, R. and Knepper, M. A. (2006) Discovery of urinary biomarkers. Mol. Cell. Proteomics. 5, 1760-1771.

27 Whitelegge, J. P., Laganowsky, A., Nishio, J., Souda, P., Zhang, H. and Cramer, W. A. (2006) Sequencing covalent modifications of membrane proteins. J. Exp. Bot. 57, 1515-1522.

28 Whitelegge, J., Halgand, F., Souda, P. and Zabrouskov, V. (2006) Top-down mass spectrometry of integral membrane proteins. Expert. Rev. Proteomics. 3, 585-596.

29 Zabrouskov, V. and Whitelegge, J. P. (2007) Increased coverage in the transmembrane domain with activated-ion electron capture dissociation for top-down Fourier-transform mass spectrometry of integral membrane proteins. J. Proteome Res. 6, 2205-2210.

30 Geels, R. B., van der Vies, S. M., Heck, A. J. and Heeren, R. M. (2006) Electron capture dissociation as structural probe for noncovalent gas-phase protein assemblies. Anal. Chem. 78, 7191-7196.

31 Xie, Y., Zhang, J., Yin, S. and Loo, J. A. (2006) Top-down ESI-ECD-FT-ICR mass spectrometry localizes noncovalent protein-ligand binding sites. J. Am. Chem. Soc. 128, 1443214433.

32 Savitski, M. M., Nielsen, M. L. and Zubarev, R. A. (2006) ModifiComb, a new proteomic tool for mapping substoichiometric post-translational modifications, finding novel types of modifications, and fingerprinting complex protein mixtures. Mol. Cell. Proteomics. 5, 935-948.

33 Roth, M. J., Forbes, A. J., Boyne, M. T., 2nd, Kim, Y. B., Robinson, D. E. and Kelleher, N. L. (2005) Precise and parallel characterization of coding polymorphisms, alternative splicing, and modifications in human proteins by mass spectrometry. Mol. Cell. Proteomics. 4, 10021008.

34 Whitelegge, J. P., Zabrouskov, V., Halgand, F., Souda, P., Bassilian, S., Yan, W., Wolinsky, L., Loo, J. A., Wong, D. T. and Faull, K. F. (2007) Protein-Sequence Polymorphisms and Post-translational Modifications in Proteins from Human Saliva using Top-Down Fouriertransform Ion Cyclotron Resonance Mass Spectrometry. Int. J. Mass Spectrom. 268, 190-197. 
35 Graciet, E., Gans, P., Wedel, N., Lebreton, S., Camadro, J. M. and Gontero, B. (2003) The small protein CP12: a protein linker for supramolecular complex assembly. Biochemistry. 42, 8163-8170.

36 Chen, Y. H., Yang, J. T. and Chau, K. H. (1974) Determination of the helix and beta form of proteins in aqueous solution by circular dichroism. Biochemistry. 13, 3350-3359.

37 Bradford, M. M. (1976) A rapid and sensitive method for the quantitation of microgram quantities of protein utilizing the principle of protein-dye binding. Anal. Biochem. 72, 248-254.

38 Loo, J. A. (2000) Electrospray ionization mass spectrometry: a technology for studying noncovalent macromolecular complexes. Int. J. Mass Spectrom. 200, 175-186.

39 Kaltashov, I. A., Zhang, M., Eyles, S. J. and Abzalimov, R. R. (2006) Investigation of structure, dynamics and function of metalloproteins with electrospray ionization mass spectrometry. Anal. Bioanal. Chem. 386, 472-481.

$40 \quad$ Rulisek, L. and Vondrasek, J. (1998) Coordination geometries of selected transition metal ions $(\mathrm{Co} 2+, \mathrm{Ni} 2+, \mathrm{Cu} 2+, \mathrm{Zn} 2+, \mathrm{Cd} 2+$, and $\mathrm{Hg} 2+)$ in metalloproteins. J. Inorg. Biochem. 71, $115-$ 127.

41 Halgand, F. and Laprevote, O. (2001) Mean charge state and charge state distribution of proteins as structural probes. An electrospray ionisation mass spectrometry study of lysozyme and ribonuclease A. Eur. J. Mass Spectrom. 7, 433-439.

42 Armitage, P. (1971) Statistical methods in medical research. . chap 7.- p 504. Blackwell Scientific Publication Oxford and Edinburgh.

43 Turecek, F. (2007) Copper-biomolecule complexes in the gas phase. The ternary way. Mass Spectrom. Rev. 26, 563-582.

44 Gardebien, F., Thangudu, R. R., Gontero, B. and Offmann, B. (2006) Construction of a 3D model of CP12, a protein linker. J. Mol. Graph. Model. 25, 186-195.

45 Gabelica, V. and De Pauw, E. (2005) Internal energy and fragmentation of ions produced in electrospray sources. Mass Spectrom. Rev. 24, 566-587.

46 Howard, T. P., Metodiev, M., Lloyd, J. C. and Raines, C. A. (2008) Thioredoxinmediated reversible dissociation of a stromal multiprotein complex in response to changes in light availability. Proc. Natl. Acad. Sci. US A. 105, 4056-4061.

47 Millhauser, G. L. (2007) Copper and the prion protein: methods, structures, function, and disease. Annu. Rev. Phys. Chem. 58, 299-320.

48 Zheng, R., Jenkins, T. M. and Craigie, R. (1996) Zinc folds the N-terminal domain of HIV-1 integrase, promotes multimerization, and enhances catalytic activity. Proc. Natl. Acad. Sci. U S A. 93, 13659-13664.

49 Uversky, V. N., Li, J. and Fink, A. L. (2001) Metal-triggered structural transformations, aggregation, and fibrillation of human alpha-synuclein. A possible molecular NK between Parkinson's disease and heavy metal exposure. J. Biol. Chem. 276, 44284-44296.

50 Roepstorff, P. and Fohlman, J. (1984) Proposal for a common nomenclature for sequence ions in mass spectra of peptides. Biomed. Mass. Spectrom. 11, 601. 
Table 1: Circular dichroism data of wild-type CP12 and mutants recorded with increasing amounts of copper. $\alpha$-helix content in $\%$ is given for each copper concentration.

\begin{tabular}{|c|c|c|c|}
\hline$\left[\mathbf{C u}^{\mathbf{2 +}}\right]$ & $\mathbf{0} \boldsymbol{\mu M}$ & $\mathbf{2 0} \boldsymbol{\mu M}$ & $\mathbf{1 0 0} \boldsymbol{\mu M}$ \\
\hline $\mathbf{W T}$ & 29 & 32 & 31 \\
\hline $\mathbf{E 2 0 A}$ & 25 & 25 & 25 \\
\hline $\mathbf{E 4 1 A}$ & 26 & 26 & 26 \\
\hline $\mathbf{E 4 2 A}$ & 23 & 23 & 23 \\
\hline $\mathbf{E 4 1 \mathbf { A }} / \mathbf{E 4 2 A}$ & 17 & 17 & 17 \\
\hline $\mathbf{E 4 1 K} / \mathbf{E 4 2 K}$ & 19 & 18 & 18 \\
\hline
\end{tabular}

Table 2: Summary of the CP12 mutations effects upon copper binding properties by Cu-IMAC experiments. Symbols: $(+),+$ and ++ reflect the proportion of CP12 detected in each step; corresponds to absence of CP12 detection; "Not retained" corresponds to elution of CP12 in the void volume of the column.

\begin{tabular}{|c|c|c|c|}
\hline CP12 & $\begin{array}{c}\text { Washing } \\
\text { steps }\end{array}$ & $\begin{array}{c}\text { 1rst } \\
\text { elution: } \\
\left(\mathrm{NH}_{4} \mathrm{OH} 0.3 \mathrm{M}\right)\end{array}$ & $\begin{array}{c}\mathbf{2}^{\text {nd }} \\
\text { elution: } \\
\text { (EDTA 1 mM) }\end{array}$ \\
\hline WT & - & ++ & + \\
\hline E18A & + & ++ & - \\
\hline E18K & ++ & + & - \\
\hline E39A & ++ & - & - \\
\hline E39K & ++ & -+ & - \\
\hline E40A & - & ++ & - \\
\hline E40K & Not retained & - & - \\
\hline E39A / E40A & ++ & - & - \\
\hline E39K/ E40K & ++ & - & - \\
\hline
\end{tabular}

Table 3: Calculated Zav values between the free and copper complexed states of each protein. $\Delta$ mutation stands for the difference between the mean Zav of the wild-type and mutant protein, $\Delta$ copper stands for the difference between the mean Zav of the free and copper complexed state of each protein. Shown are the means \pm S.D. of 4 measurements.

\begin{tabular}{|c|c|c|c|c|}
\hline CP12 & Mean Zav & $\Delta$ mutation & $\begin{array}{c}\text { Mean Zav } \\
\text { with copper }\end{array}$ & $\Delta$ copper \\
\hline WT & $5.51 \pm 0.38$ & & $5.51 \pm 0.38$ & $0 \pm 0.27$ \\
\hline E20A & $5.62 \pm 0.38$ & $0.11 \pm 0.27$ & $5.09 \pm 0.26$ & $-0.53 \pm 0.23$ \\
\hline E41A & $5.76 \pm 0.41$ & $0.25 \pm 0.28$ & $5.45 \pm 0.37$ & $-0.31 \pm 0.28$ \\
\hline E42A & $5.64 \pm 0.40$ & $0.13 \pm 0.28$ & $5.08 \pm 0.24$ & $-0.56 \pm 0.23$ \\
\hline E41A / E42A & $5.52 \pm 0.39$ & $0.01 \pm 0.27$ & $5.16 \pm 0.29$ & $-0.36 \pm 0.24$ \\
\hline E41K/ E42K & $6.08 \pm 0.41$ & $0.57 \pm 0.28$ & $5.79 \pm 0.40$ & $-0.29 \pm 0.29$ \\
\hline
\end{tabular}




\section{Figure legends:}

Figure 1: (A) Ribbon and (B) surface structures of CP12 showing the location of the Glu residues E20, E41 and E42. Pymol software (http://pymol.sourceforge.net/) was used to visualize the CP12 protein structure. These analyses confirm the accessibility of selected residues, E20, and E41 and E42 in the highly conserved sequence $\mathrm{V}_{40}$ EESLAA $_{46}$ to bind copper.

Figure 2: (A) CD spectra of $10 \mu \mathrm{M}$ wild-type and mutant CP12s (curves 1 to 6 ) and of wild-type CP12 incubated with $\mathrm{Cu}^{2+}$ (curves 7 to 8). Spectra were obtained in $10 \mathrm{mM}$ phosphate buffer $\mathrm{pH}$ 6.0. (B) Fluorescence spectra of $1.8 \mu \mathrm{M}$ wild-type and mutant CP12 in $50 \mathrm{mM}$ Tris, $100 \mathrm{mM}$ $\mathrm{NaCl}$ at $\mathrm{pH} 8$ buffer $\left(\lambda_{\mathrm{exc}}=290 \mathrm{~nm} ; \lambda_{\mathrm{em}}=300-450 \mathrm{~nm}\right)$.

Figure 3: Comparison of MS spectra of mutant CP12 proteins recorded under non denaturing conditions without and with copper. (A) Free E20A (upper panel) and copper complexed E20A (lower panel). B) Free E41K/E42K (upper panel) and copper complexed E41K/E42K (lower panel), peaks corresponding respectively to the free state and $E 41 \mathrm{~K} / \mathrm{E} 42 \mathrm{~K} / \mathrm{Cu}^{2+}$ complex state are labeled with $\mathrm{x}$ and $\bullet$. $\mathrm{Z}$ stands for charge states of the peaks.

Figure 4: A) MSMS spectra of the wild-type CP12 without (upper panel) and with copper (lower panel). B) MSMS spectra of the E41K/E42K mutant without (upper panel) and with copper (lower panel). Fragment ions bearing a copper ion are labeled with an asterisk. Z stands for charge states of the peaks.

Figure 5: A) Sequence charts of wild-type CP12 and mutants, showing fragment ions generated by top-down MSMS experiments in the presence of copper. An illustration of the fragmentation nomenclature is given in the insert (top right) [50]. The bold brackets $(7)$ represent fragment ions bearing copper ion. Asterisk denotes b70 ions that correspond to disulfide bond breakage. These ions are only observed when CP12 is complexed with copper. The mutation positions are in bold and underlined. B) Location on CP12 sequence of the three regions proposed to be involved in copper binding (Region $1=\mathrm{D} 16$ to $\mathrm{D} 23$; region $2=\mathrm{D} 38$ to $\mathrm{K} 50$; region $3=\mathrm{D} 70$ to E76). The $\alpha$-helices are depicted in gray boxes, and positively and negatively charged residues are bold. The residues that have been mutated in this study are indicated by arrows. 


\section{Figure 1:}

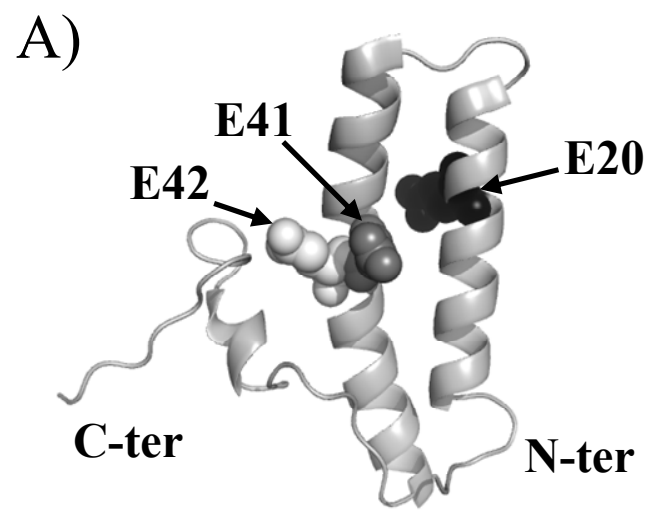

B)

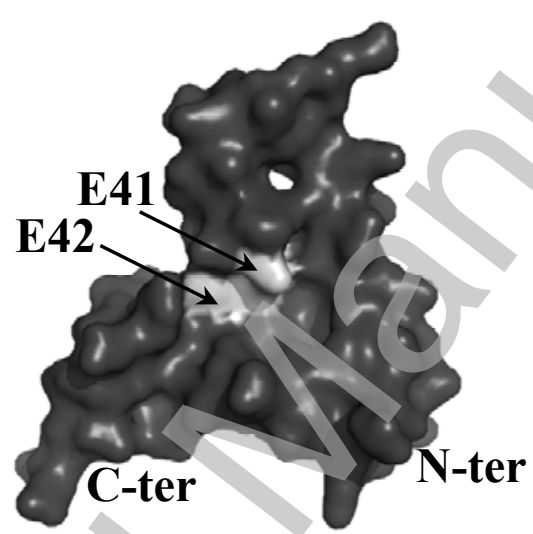




\section{Figure 2:}
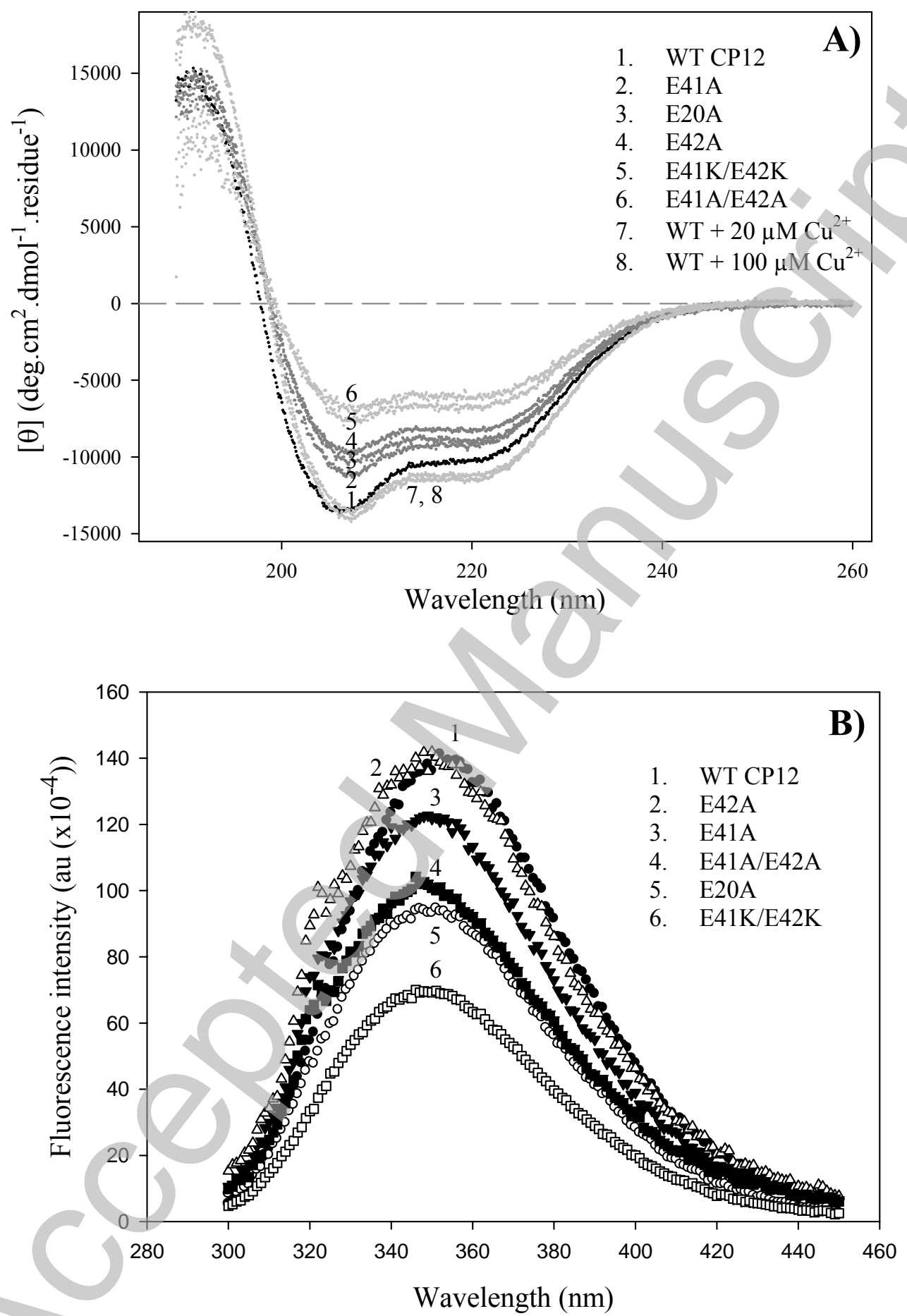


\section{Figure 3:}

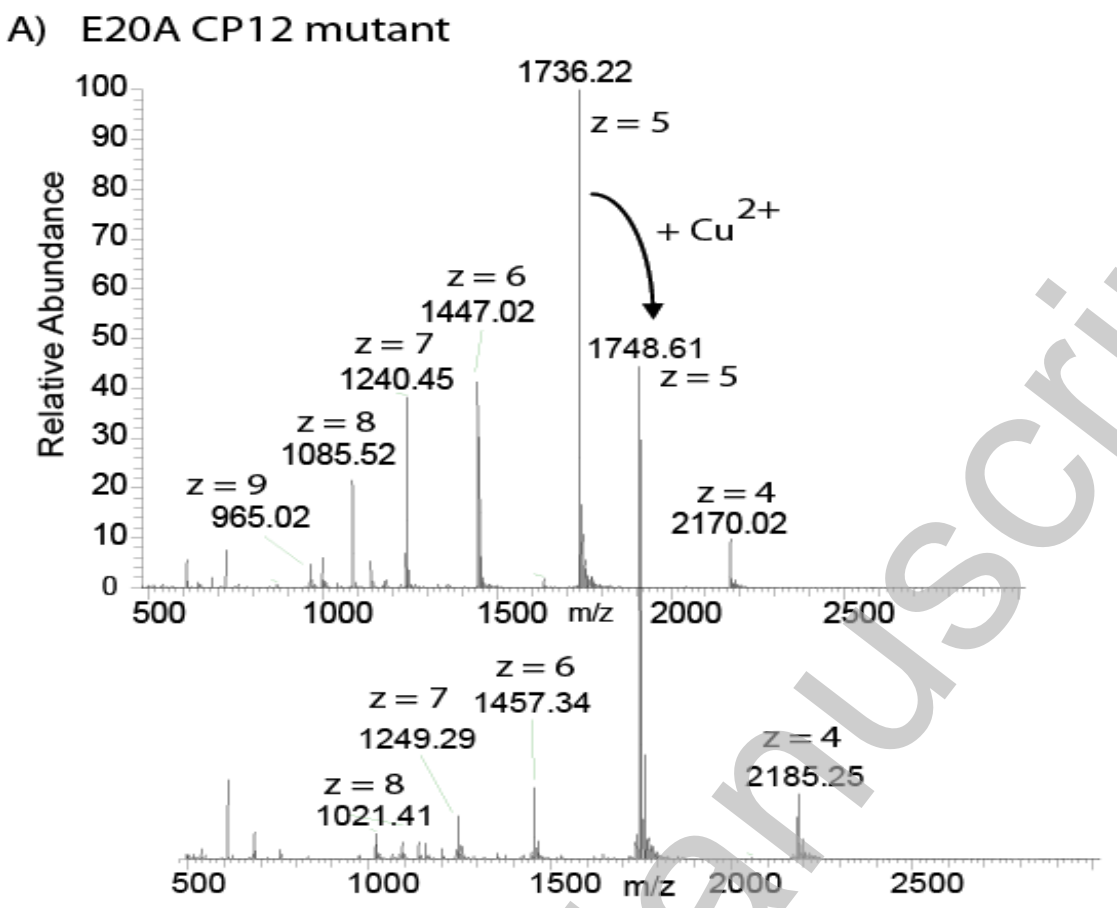

B) $\mathrm{E} 41 \mathrm{~K} / \mathrm{E} 42 \mathrm{~K}$ double CP12 mutant

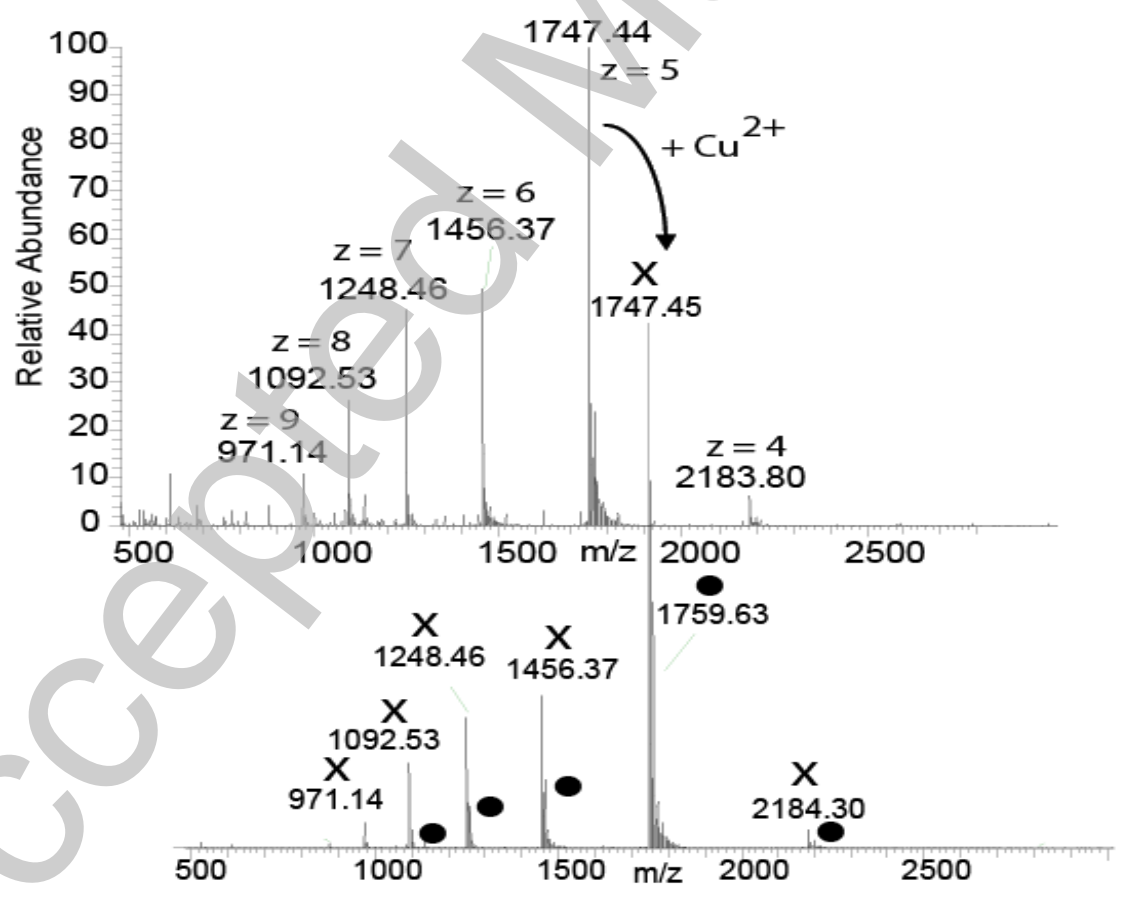




\section{Figure 4:}

A) WT
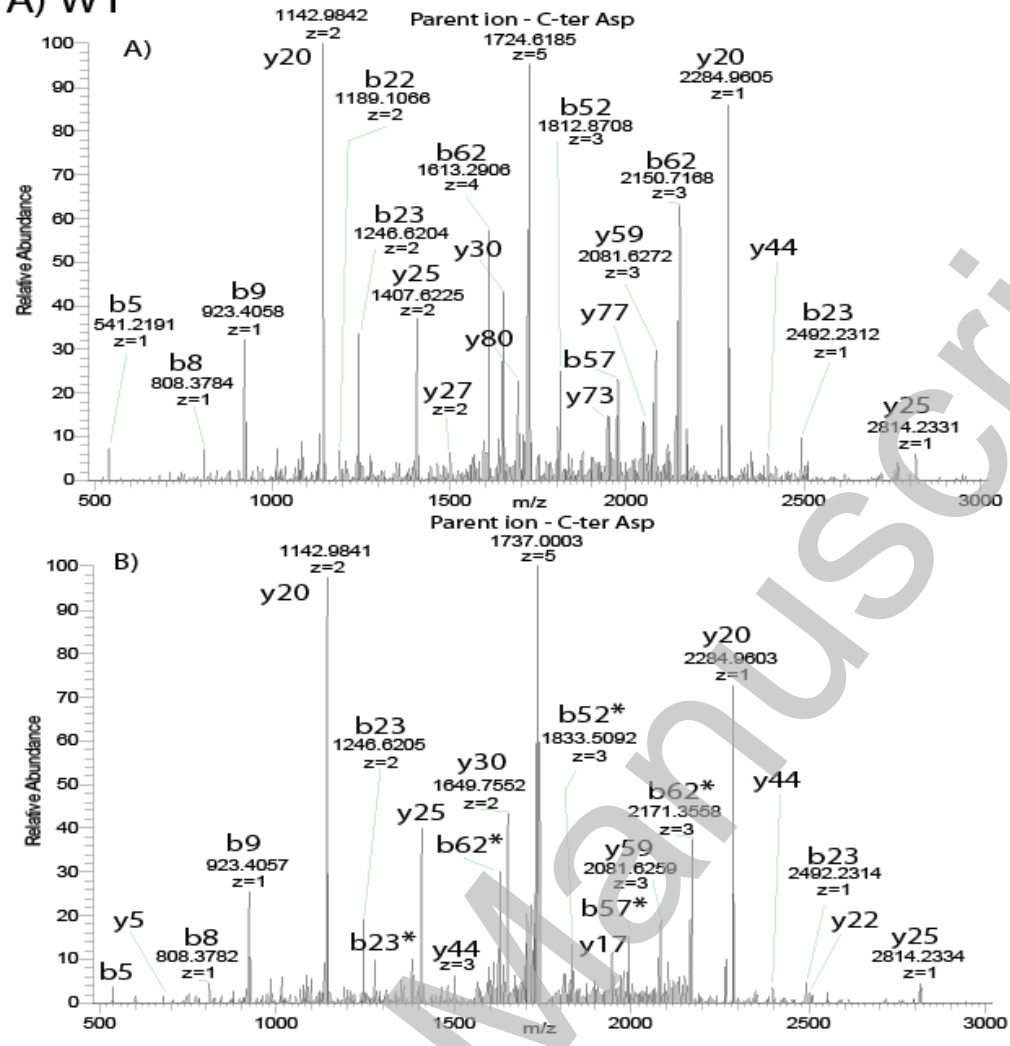

B) E41K/ E42K Parention - c-ter Asp

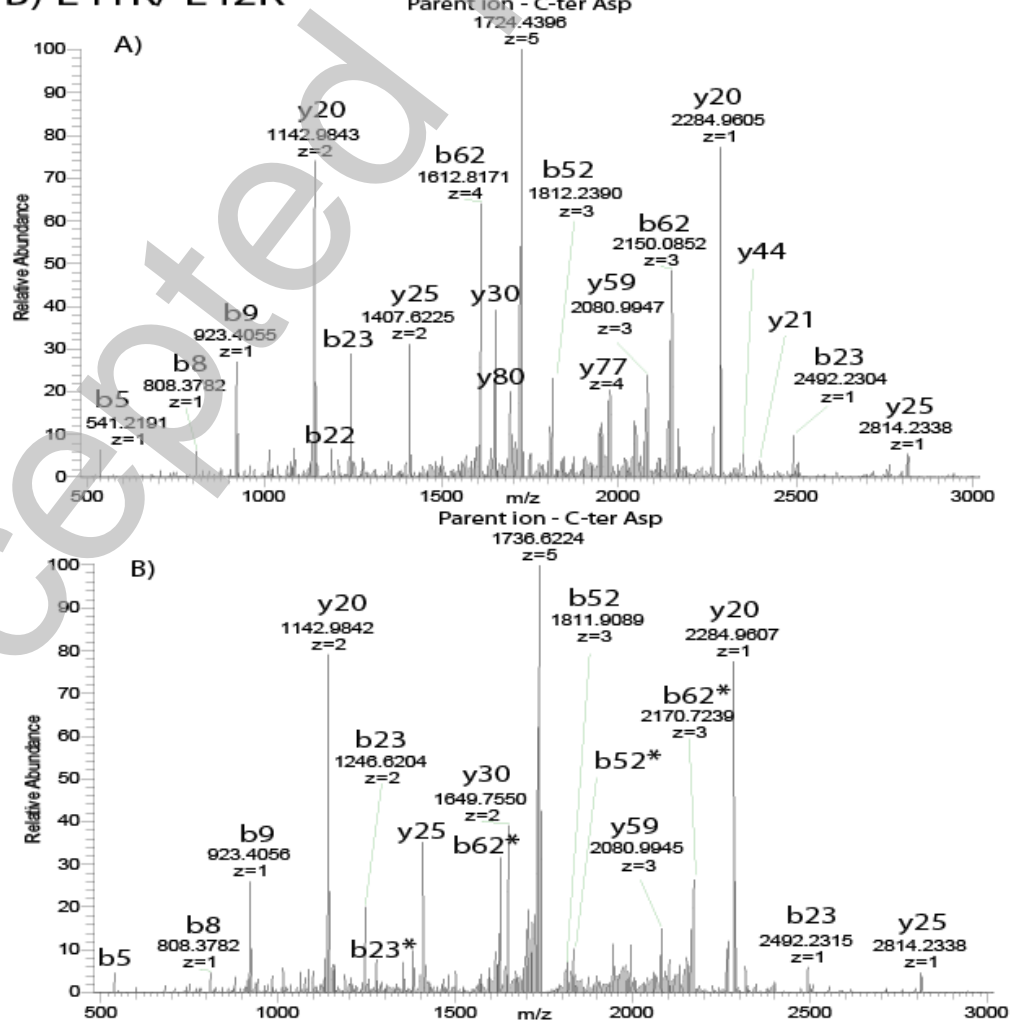




\section{Figure 5:}

A)

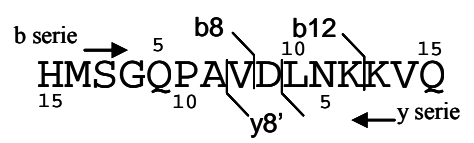

WT

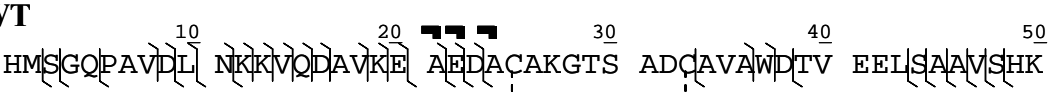

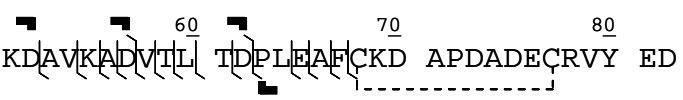

E20A $10 \quad 70 \quad 20 \quad 79 \quad 30 \quad 40 \quad 50$

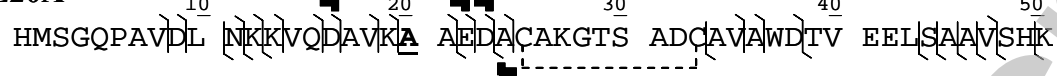

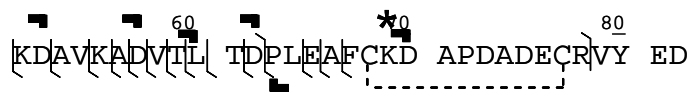

E41A $10 \quad 20 \quad 79030 \quad 30 \quad 40$

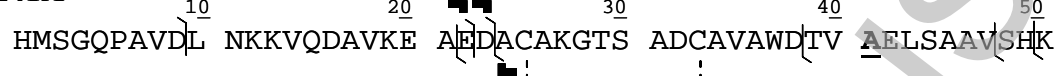

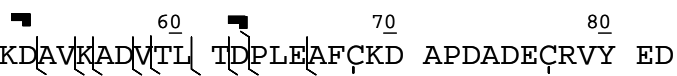

E42A

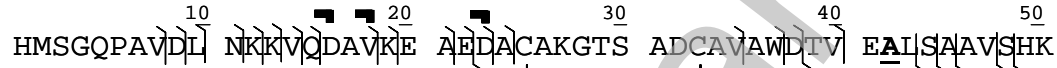
KDDAVLADLVTL TDPLLEAFCYKD APDADECRVY ED E41A / E42A
HMSGQPAVDLLI
10

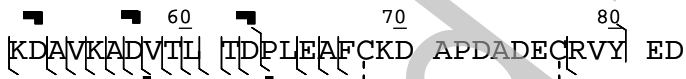

E41K/ E42K ${ }_{10} 20 \quad 30 \quad 40$

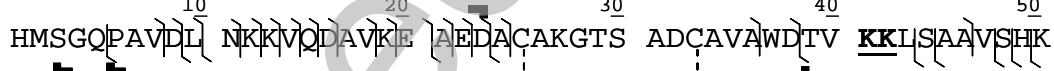

- 2

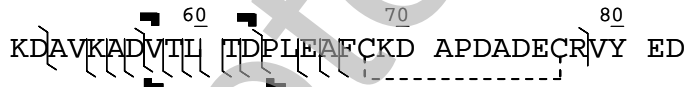

B)

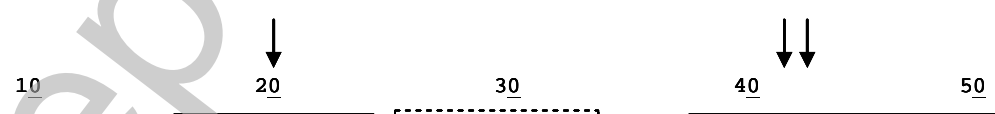

HMSGQPAVDL NKKVQDAVKE AEDACAKGTS ADCAVAWDTV EELSAAVSHK

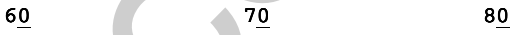

KDAVKADVTL TDPLEAFCKD APDADECRVY ED 